\title{
Differential clinical manifestations and clinical outcome of cancer-related pulmonary embolism
}

\author{
Min Sun Kim ${ }^{1,}$, Haseong Chang ${ }^{1,}$, Su Yeon Lee ${ }^{1}$, Sun Hye Shin ${ }^{2}$, HyeYun Park ${ }^{2}$, Sung-A Chang ${ }^{1}$, \\ Taek Kyu Park ${ }^{1}$, Duk-Kyung Kim ${ }^{1}$, and Eun Kyoung Kim ${ }^{1}$
}

\begin{abstract}
${ }^{1}$ Division of Cardiology, Department of Medicine, Heart Vascular Stroke Institute, ${ }^{2}$ Division of Pulmonology, Department of Medicine, Samsung Medical Center, Sungkyunkwan University School of Medicine, Seoul, Korea
\end{abstract}

Received: July 17, 2018

Revised : September 7, 2018

Accepted: November 11, 2018

\section{Correspondence to}

Eun Kyoung Kim, M.D.

Division of Cardiology, Department of Medicine, Heart Vascular Stroke Institute, Samsung Medical Center, Sungkyunkwan University School of Medicine, 81 Irwon-ro, Gangnam-gu, Seoul o6351, Korea

Tel: $+82-2-3410-3419$

Fax: $+82-2-3410-3849$

E-mail: ekbobi.kim@samsung.com

*These authors contributed equally to this work.
Background/Aims: Although acute pulmonary embolism (PE) adversely impacts survival and should be treated regardless of cancer, the treatment rate of cancer-related PE is relatively low. We aimed to compare clinical characteristics and long term prognosis of $\mathrm{PE}$ in patients with or without cancer.

Methods: From March 2010 to December 2013, patients with newly diagnosed PE were analyzed. Baseline demographics, comorbidities, cancer status and clinical manifestations of PE were recorded. We defined primary composite outcome as recurrent venous thromboembolism (VTE) and death from PE.

Results: Among a total of 976 patients with PE, the 703 (72.0\%) had cancer-related PE. Cancer-related PE group was more frequently asymptomatic ( $54.5 \%$ vs. $13.2 \%$, $p<0.001$ ), less extensive (involvement of bilateral pulmonary arteries: $42.8 \%$ vs. $51.3 \%, p=0.017$; lung infarction: $5.3 \%$ vs. $10.3 \%, p=0.005$ ) and less likely to accompany right ventricular dysfunction (10.3\% vs. $27.2 \%, p<0.001)$ compared with the non-cancer PE group. Anticoagulation was less frequently underwent in patients with cancer-related PE than those without cancer (62.0\% vs. 81.7\%, $p<0.001)$. A composite of recurrent VTE and death from PE was significantly higher in the cancer-related PE group (14.4\% vs. $6.6 \%, p=0.001$ ).

Conclusions: Although PE in cancer patients were seem to be less aggressive initially, compared to those without cancer, they had significantly poor prognosis. Given a high rate of recurrent VTE and relatively similar risk of anticoagulation associated bleeding events in cancer patients, more active treatment of PE is warranted in cancer patients.

Keywords: Pulmonary embolism; Venous thromboembolism; Neoplasms

\section{INTRODUCTION}

The prevalence of venous thromboembolism (VTE) in patients with active cancer has been reported as four to seven times higher than those without cancer and is increasing with cancer status $[1,2]$. This is because (1) cancer patients have increased thrombotic risk due to the malignancy itself or chemotherapy, (2) cancer patients frequently undergo serial computed tomography (CT) scanning, which enables early detection of pulmonary embolism (PE). With the increased use of various anticancer drugs that are potentially thrombogenic, the incidence of VTE continues to increase [3-6]. Because the pathogenesis of VTE in cancer patients is quite different from VTE of other cause, such as thrombophilia, trauma, or immobilization, the clinical progress of cancer-related VTE is also different. However, available data regarding the differential clinical manifestations of 
cancer-related VTE are limited.

Although current guidelines for PE management uniformly recommend that active cancer patients should receive anticoagulation regardless of the location and extent of $\mathrm{PE}[7,8]$, the treatment rate of cancer-related $\mathrm{PE}$ in real world practice is quite low [9]. Concerns about high bleeding tendency or potential interactions between anticoagulants and anticancer drugs in patients with advanced stage cancer cause physicians to neglect to treat PE. However, given that the expected survival increasing in patients with active cancer, it is important to treat combined morbidities such as deep vein thrombosis (DVT) and PE. In the present study, we identified differential clinical manifestations and prognosis of cancer-related PE compared to PE of other causes.

\section{METHODS}

\section{Study population and design}

The study cohort consisted of consecutive patients diagnosed with PE between October 2010 and December 2013 in our clinic. Among 1,164 eligible patients, patients with known PE before visiting our clinic $(\mathrm{n}=58)$, diagnosed with PE by abdomen CT scan that partially covered the pulmonary artery $(n=55)$, with filling defect in pulmonary artery stump site after cancer operation $(\mathrm{n}=$ 21), who were lost to follow-up $(\mathrm{n}=2 \mathrm{O})$, and who were finally diagnosed with tumor emboli $(n=19)$ were excluded. After additional exclusion of 15 patients with chronic PE, 976 patients were included in final analysis (Fig. 1). Clinical, laboratory, echocardiography, CT findings of $\mathrm{PE}$, and underlying cancer status were collected from our PE registry. The local Institutional Review Board of Samsung Medical Center (2017-12-073) approved this study and waived the requirement for written informed consent.

Provoking factors causing PE were evaluated and, classified as active cancer, major surgery within three months of index event that required general anesthesia lasting 30 minutes, immobilization defined as absolute bed rest for 5 consecutive days and airplane flight longer than six hours, pregnancy or puerperium at the time of $\mathrm{PE}$, usage of oral contraceptives or hormonal replacement therapy, or trauma. PE was considered 'unprovoked' in the absence of the above factors. Unprovoked

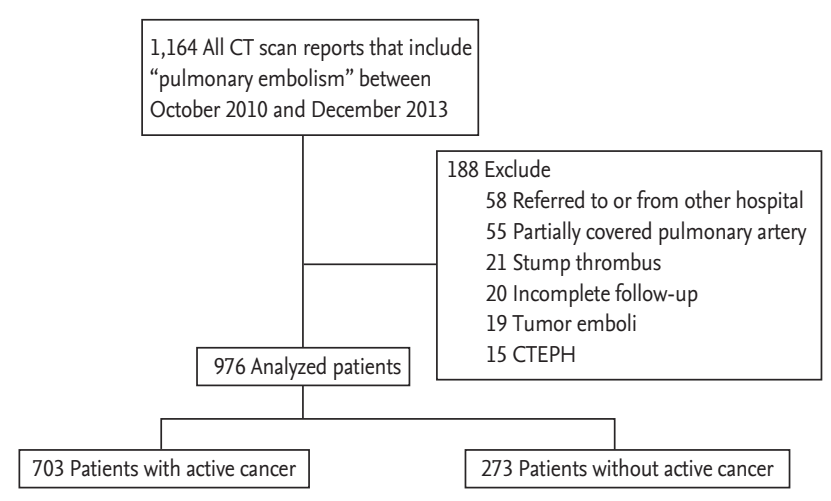

Figure 1. Flow chart of eligible patients. Among 1,164 cases of newly diagnosed pulmonary embolism (PE) on computed tomography (CT) scan between October 2010 and December 2013, a total of 976 eligible cases were identified. Seven hundred and three patients had active cancer at the time of detection of PE. CTEPH, chronic thromboembolic pulmonary hypertension.

PE was classified as hereditary thrombophilia, antiphospholipid syndrome, or idiopathic event. Patients were classified as having cancer-related PE when they had recurrent or progressive cancer or any malignancy that required curative or palliative treatment at the time of PE detection. Both solid and hematologic malignancies were eligible.

On CT imaging, PE was defined as the existence of contrast filling defects in one or more branches of the pulmonary artery. Location of PE and associated lung infarction was assessed. Proximal PE was defined as PE involving main or lobar level of pulmonary arteries on CT imaging. Distal PE was defined as PE involving segmental or subsegmental level of the pulmonary arteries. Lower extremity DVT was confirmed using Doppler ultrasonography or CT. Proximal DVT was defined as occurring in the popliteal vein and above. Presence of right ventricular (RV) dysfunction was assessed using two-dimensional echocardiography.

\section{Clinical outcomes and definitions}

The primary outcome of this study was a composite of recurrent DVT/PE and PE-related death. Secondary end points included all cause of death, which was considered to be a cardiac cause unless a definite non-cardiac cause was established. Clinically significant major and minor bleeding events during anticoagulation were also assessed. Major bleeding was defined according to the In- 
Table 1. Baseline characteristics

\begin{tabular}{|c|c|c|c|c|}
\hline Characteristic & PE without cancer $(n=273)$ & PE with cancer $(\mathrm{n}=703)$ & $\operatorname{Total}(n=976)$ & $p$ value \\
\hline Age, yr & $69(54-77)$ & $63(55-71)$ & $65(54-73)$ & $<0.001$ \\
\hline Male sex & $122(44 \cdot 7)$ & $397(56.5)$ & $519(53.2)$ & 0.001 \\
\hline $\mathrm{BMI}, \mathrm{kg} / \mathrm{m}^{2}$ & $23.8(21.0-26.7)$ & $23.2(21.2-25.4)$ & $23.5(21.1-25.6)$ & 0.016 \\
\hline Current smoker & $33(12.1)$ & $47(6.7)$ & $80(8.2)$ & 0.006 \\
\hline \multicolumn{5}{|l|}{ Comorbidities } \\
\hline Diabetes & $40(14.7)$ & $103(14.7)$ & $143(14.7)$ & 1.000 \\
\hline Hypertension & $102(37 \cdot 4)$ & $235(33 \cdot 4)$ & $337(34 \cdot 5)$ & 0.246 \\
\hline Chronic kidney disease & $25(9.2)$ & $39(5 \cdot 5)$ & $64(6.6)$ & 0.041 \\
\hline Previous DVT & $15(5 \cdot 5)$ & $24(3.4)$ & $39(4.0)$ & 0.136 \\
\hline
\end{tabular}

Values are presented as median (interquartile range) or number (\%).

$\mathrm{PE}$, pulmonary embolism; BMI, body mass index; DVT, deep vein thrombosis.

ternational Society on Thrombosis and Hemostasis criteria as fatal bleeding or clinically overt bleeding together with a decrease in hemoglobin level of at least $2 \mathrm{~g} / \mathrm{dL}$ or transfusion of at least 2 units of packed red blood cells occurring at a critical site (intracranial, intraspinal, intraocular, retroperitoneal, pericardial, intraarticular, and intramuscular with compartment syndrome) [10]. All non-major bleeding was considered minor bleeding.

\section{Statistical analyses}

Baseline characteristics are summarized with continuous variables and expressed as mean \pm standard deviation or median with interquartile range (IQR). Categorical data are presented as a percentage and the number of events. Continuous variables were analyzed using an independent $t$ test or Wilcoxon rank sum test as appropriate, and categorical variables were analyzed using a chi-square test or Fisher's exact test.

Event-free survival curves were constructed using the Kaplan-Meier estimator and compared using log-rank tests. Cox proportional-hazards regression with backward elimination was used to estimate the hazard ratio as a measure of the relative risk of $\mathrm{PE}$ among cancer patients compared with non-cancer patients adjusting for baseline characteristics and comorbidity. All analysis was conducted with SPSS software version 23.0 (IBM Co., Armonk, NY, USA). Statistical significance was concluded at a 2-sided significance level of 0.05 for all analyses.

\section{RESULTS}

Among 976 patients who were finally analyzed (age: 65 years [IQR, 54 to 73]; 53.2\% male), 703 (72.0\%) were classified to the cancer-related PE group. Lung cancer (44.8\%) was the most prevalent, followed by colon cancer (8.7\%), gynecological malignancy (7.3\%), and lymphoma (5.8\%). Most patients had advanced stage cancer with distant metastasis (68.4\%). Among 273 patients (28.0\%) with non-cancer-related PE, 192 patients had provoked PE, which was commonly caused by immobilization (33.0\%) or surgery (23.1\%). Table 1 shows the baseline characteristics. Patients with cancer-related PE were more frequently male, less commonly smokers, and younger than those with non-cancer PE (male: $56.5 \%$ vs. $44.7 \%$, p $=0.001$; smoker: $6.7 \%$ vs. $12.1 \%, p=0.006$; age: 63 years [IQR, 55 to 71] vs. 69 years [IQR, 54 to 77], $p<0.001$ ). There were no significant differences in pre-existing diabetes, hypertension, or DVT between two groups except the presence of chronic kidney disease (5.5\% vs. $9.2 \%, p=$ 0.041).

Table 2 shows the initial clinical manifestation of $\mathrm{PE}$ in both groups. Cancer-related PE group was more frequently asymptomatic compared to non-cancer $\mathrm{PE}$ group ( $54.5 \%$ vs. $13.2 \%, p<0.001$ ). Critical vital status at the time of PE diagnosis, including hypoxia, shock, and tachycardia, was significantly less severe in patients with cancer-related PE than in those without cancer (all $p<$ 0.05). PE-related cardiac distress was also less frequent in patients with cancer-related PE (RV dysfunction: 
Table 2. Clinical manifestation of PE

\begin{tabular}{|c|c|c|c|c|}
\hline Characteristic & PE without cancer $(n=273)$ & PE with cancer $(\mathrm{n}=703)$ & $\operatorname{Total}(n=976)$ & $p$ value \\
\hline \multicolumn{5}{|l|}{ Initial symptom/sign } \\
\hline No symptom & $36(13.2)$ & $383(54.5)$ & $419(42.9)$ & $<0.001$ \\
\hline Dyspnea & $139(50.9)$ & $201(28.6)$ & $340(34.8)$ & $<0.001$ \\
\hline Chest pain & $41(15.0)$ & $32(4.6)$ & $73(7.5)$ & $<0.001$ \\
\hline Leg swelling & $47(17.2)$ & $65(9.2)$ & $112(11.5)$ & $<0.001$ \\
\hline Fever & $50(18.3)$ & $40(5.7)$ & $90(9.2)$ & $<0.001$ \\
\hline Hypoxia & $134(50.0)$ & $151(22.3)$ & $285(30.2)$ & $<0.001$ \\
\hline Tachycardia & $114(42.5)$ & $233(34.4)$ & $347(36.7)$ & 0.020 \\
\hline Shock & $35(13.1)$ & $36(5 \cdot 3)$ & $71(7.5)$ & $<0.001$ \\
\hline Unexpected $\mathrm{PE}^{\mathrm{a}}$ & $40(14.7)$ & $474(67.4)$ & $514(52.7)$ & $<0.001$ \\
\hline \multicolumn{5}{|l|}{ Laboratory finding } \\
\hline D-dimer & $5.9(2.8-15.4)$ & $9.5(4.7-19.1)$ & $8.0(3.7-18.0)$ & $<0.001$ \\
\hline Hemoglobin & $12.1(10.0-13.4)$ & $11.2(10.1-12.6)$ & $11.4(10.0-13.0)$ & 0.001 \\
\hline Platelet & $197(152-250)$ & $203(141-270)$ & $200(144-264)$ & 0.751 \\
\hline NT-proBNP & $900(144-3,625)$ & $436(183-1,630)$ & $586(167-2,502)$ & 0.028 \\
\hline CK-MB & $1.97(0.78-4.75)$ & $1.56(0.57-3.32)$ & $1.83(0.69-3.91)$ & 0.024 \\
\hline Troponin I & $0.029(0.006-0.132)$ & $0.019(0.006-0.115)$ & $0.022(0.006-0.118)$ & 0.145 \\
\hline \multicolumn{5}{|c|}{ Echocardiographic finding } \\
\hline RV dysfunction & $41(27.2)$ & $17(10.3)$ & $58(18.4)$ & $<0.001$ \\
\hline D-shaped LV & $32(21.2)$ & $14(8.5)$ & $46(14.6)$ & 0.001 \\
\hline \multicolumn{5}{|l|}{ CT finding } \\
\hline Proximal $^{\mathrm{b}}$ & $170(62.3)$ & $415(59.0)$ & $585(59.9)$ & 0.354 \\
\hline Bilateral involvement & $140(51.3)$ & $301(42.8)$ & $441(45.2)$ & 0.017 \\
\hline Lung infarction & $28(10.3)$ & $37(5 \cdot 3)$ & $65(6.7)$ & 0.005 \\
\hline Coexisting DVT ${ }^{\mathrm{c}}$ & $139(60.2)$ & $160(57.6)$ & $299(58.7)$ & 0.550 \\
\hline Distal DVT & $45(19.5)$ & $52(18.7)$ & $97(19.1)$ & 0.836 \\
\hline Proximal DVT & $94(40.7)$ & $108(38.8)$ & $202(39.7)$ & 0.836 \\
\hline
\end{tabular}

Values are presented as number (\%) or median (interquartile range).

PE, pulmonary embolism; NT-proBNP, N-terminal pro-blood natriuretic peptide; CK-MB, creatine kinase myocardial band; $\mathrm{RV}$, right ventricle; LV, left ventricle; CT, computed tomography; DVT, deep vein thrombosis.

${ }^{a}$ Unexpected PE was defined as incidentally detected PE on computed tomographic examination without PE-related symptom/ sign.

${ }^{\mathrm{b}}$ Proximal PE includes filling defects in the main, central, and lobar pulmonary artery.

${ }^{c}$ DVT is confirmed using Doppler ultrasonography or computed tomography. Proximal DVT was defined as occurring in the popliteal vein and above.

10.3\% vs. $27.2 \%, p<0.001$; N-terminal pro-blood natriuretic peptide: $436.0 \mathrm{pg} / \mathrm{mL}$ [IQR, 183.0 to $1,630.0$ ] vs. 900.0 pg/mL [IQR, 144.0 to 3,625.0], $p=0.028$ ). Cardiac enzymes also tended to be lower in patients with cancer-related PE. On chest CT, involvement of bilateral pulmonary arteries (42.8\% vs. $51.3 \%, p=0.017$ ) and com- bined lung infarction (5.3\% vs. 10.3\%, $p=0.005$ ) were less prevalent in patients with cancer-related $\mathrm{PE}$ than those with non-cancer PE. There was no significant difference in the incidence of coexisting DVT.

Anticoagulation was less frequent in patients with cancer-related PE than those without cancer (62.0\% vs. 
Table 3. Treatment of PE

\begin{tabular}{|c|c|c|c|c|}
\hline Characteristic & PE without cancer $(n=273)$ & PE with cancer $(n=703)$ & Total $(n=976)$ & $p$ value \\
\hline PE treatment & $223(81.7)$ & $436(62.0)$ & $659(67.5)$ & $<0.001$ \\
\hline \multicolumn{5}{|l|}{ Anticoagulation } \\
\hline VKA & $184(67.4)$ & $268(38.1)$ & $452(46.3)$ & \\
\hline LMWH & $18(6.6)$ & $156(22.2)$ & $174(17.8)$ & \\
\hline NOAC & $21(7 \cdot 7)$ & $12(1.7)$ & $33(3.4)$ & \\
\hline Thrombolysis ${ }^{\mathrm{a}}$ & $22(8.1)$ & $6(0.9)$ & $28(2.9)$ & $<0.001$ \\
\hline Thrombectomy ${ }^{\mathrm{a}}$ & $7(2.6)$ & $5(0.7)$ & $12(1.2)$ & 0.045 \\
\hline Anticoagulation duration, mon & $6.0(3.2-11.9)$ & $4.3(2.3-7.0)$ & $5.0(2.6-7.8)$ & 0.017 \\
\hline Mean follow-up duration, mon & $17.5(3.0-40.2)$ & $8.9(2.6-23.8)$ & $10.4(2.6-28.5)$ & $<0.001$ \\
\hline
\end{tabular}

Values are presented as number (\%) or median (interquartile range).

$\mathrm{PE}$, pulmonary embolism; VKA, vitamin K antagonist; LMWH, low molecular weighted heparin; NOAC, new oral anticoagulant.

${ }^{a}$ All patients who treated with thrombolysis or thrombectomy received anticoagulation therapy.

Table 4. Clinical outcome and bleeding complication

\begin{tabular}{|c|c|c|c|c|}
\hline Characteristic & PE without cancer $(n=273)$ & PE with cancer $(\mathrm{n}=703)$ & $\operatorname{Total}(\mathrm{n}=976)$ & $p$ value \\
\hline Recurrent VTE or PE death & $18(6.6)$ & $101(14.4)$ & $119(12.2)$ & 0.001 \\
\hline Recurrent VTE & $11(4.0)$ & $78(11.1)$ & $89(9.1)$ & 0.001 \\
\hline PE death & $8(2.9)$ & $23(3 \cdot 3)$ & $31(3.2)$ & 0.785 \\
\hline All death & $43(15.8)$ & $431(61.3)$ & $474(48.6)$ & $<0.001$ \\
\hline \multicolumn{5}{|l|}{ Bleeding complication } \\
\hline Major bleeding & $9(3.3)$ & $27(3.8)$ & $36(3.7)$ & 0.850 \\
\hline Minor bleeding & $6(2.2)$ & $12(1.7)$ & $18(1.8)$ & 0.601 \\
\hline
\end{tabular}

Values are presented as number (\%).

PE, pulmonary embolism; VTE, venous thromboembolism.

81.7\%, $p<0.001)$. Patients with cancer-related PE had more frequently use of vitamin $\mathrm{K}$ antagonist as anticoagulation than those with non-cancer PE. On the other hand, in the cancer-related PE group, the frequency of low molecular weighted heparin was relatively higher compared to non-cancer PE group. Details on PE treatment are provided in Table 3. Thrombolysis and surgical thrombectomy were rarely performed in patients with cancer-related PE. Among patients treated with anticoagulants, clinically relevant major or minor bleeding events were not different between the two groups (major bleeding: $3.8 \%$ vs. $3.3 \%, p=0.850$; minor bleeding: $1.7 \%$ vs. $2.2 \%, p=0.601$ ) (Table 4). During follow-up periods, a composite of recurrent VTE and death from PE was significantly higher in the cancer-related PE group (14.4\% vs. $6.6 \%, p=0.001$ ) (Fig. 2). In particular, recurrent VTE events occurred more frequently in patient with cancer-related PE (11.1\% vs. $4.0 \%, p=0.001)$. No anticoagulation and current smoking were independently associated with poor prognosis in patients with non-cancer PE, while no anticoagulation was the only prognostic factor in those with cancer-related PE (Table 5).

After excluding patients who presented with unexpected incidentally detected PE, subgroup analysis was also performed in those with symptomatic PE (229 patients with cancer-related $\mathrm{PE}$ and 233 patients with non-cancer $\mathrm{PE})$. Baseline characteristics and clinical manifestations of PE in this subgroup are shown in Supplementary Tables 1 and 2. Patients with suspected cancer-related PE consistently had a higher risk of recurrent VTE com- 

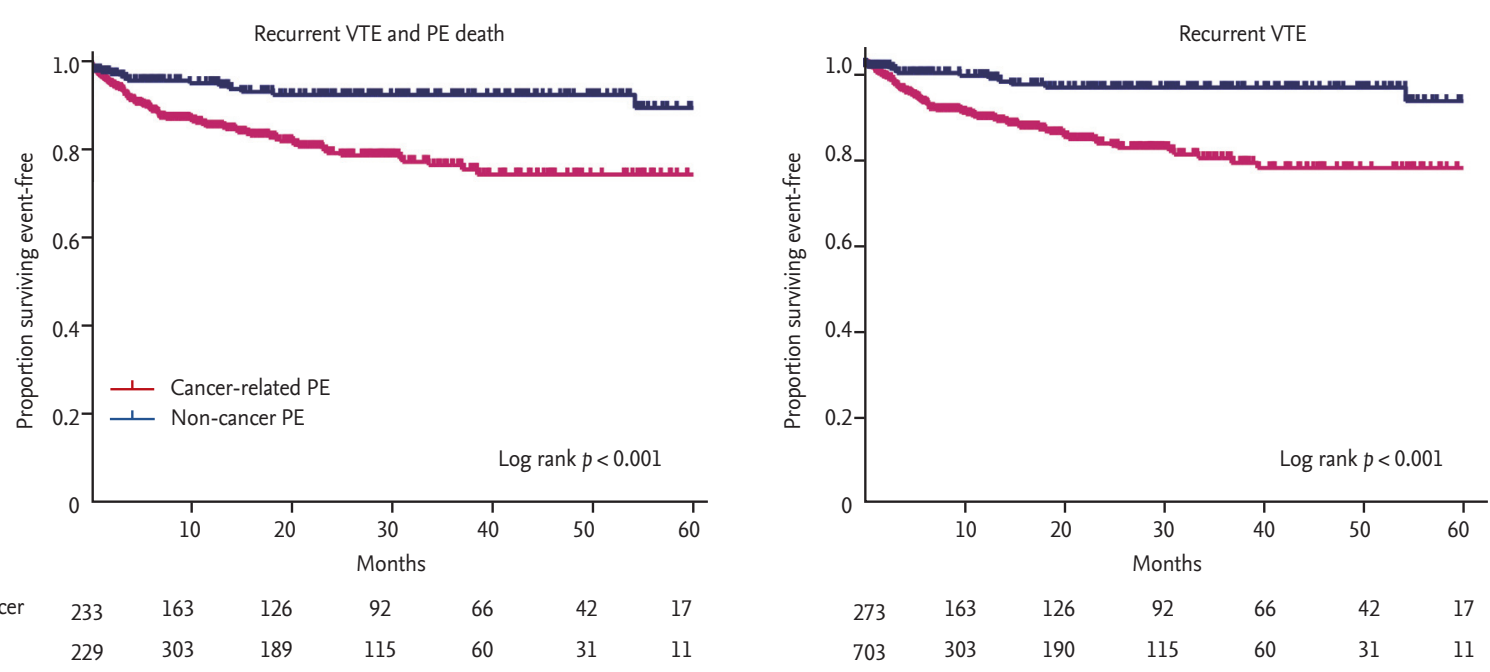

A

B

Figure 2. Clinical outcome according to the presence of cancer in patients with pulmonary embolism (PE). (A) Kaplan-Meier curve for primary composite outcome between cancer group and non-cancer group. Non-cancer group presented better prognosis than cancer group (hazard ratio, 51.11; $p<0.001$ ). (B) Kaplan-Meier curve for recurrent venous thromboembolism (VTE) only showed similar result that cancer group had more events than non-cancer group. PE, pulmonary embolism.

Table 5. Predictive factors for recurrent VTE and death from PE

\begin{tabular}{lccc}
\hline Variable & Adjusted HR & 95\% CI & $p$ value \\
\hline PE without cancer & & & 0.018 \\
$\quad$ No anticoagulation & 5.34 & $1.3-21.5$ & 0.077 \\
$\quad$ Proximal located PE & 4.63 & $0.8-25.3$ & 0.047 \\
$\quad$ Current smoking & 3.63 & $1.0-13.0$ & $<0.001$ \\
PE with cancer & & & 0.056 \\
$\quad$ No anticoagulation & 9.26 & $2.7-32.3$ & 0.068 \\
\hline Proximal located PE & 3.74 & $1.0-14.5$ & $0.9-8.6$ \\
\hline Bilateral lung involvement & 2.83 & & 0 \\
\hline
\end{tabular}

Adjusted for age, gender, body mass index, no anticoagulation, duration of treatment, proximal located PE, bilateral lung involvement, shock, tachycardia, smoking, right ventricular dysfunction, and unexpected PE.

VTE, venous thromboembolism; PE, pulmonary embolism; HR, hazard ratio; CI, confidence interval.

pared to those with suspected non-cancer PE (Fig. 3). In multivariate analysis, the presence of cancer itself was the strongest poor prognostic factor for PE irrespective of suspected PE or incidental PE (adjusted hazard ratio, 9.75; 95\% confidence interval, 2.4 to $39.2 ; p=0.001$ ) (Supplementary Table 3).

\section{DISCUSSION}

In this observational study, we investigated the clinical features and prognosis of PE according to causes that were cancer-related or not. Compared to patients with non-cancer PE, those with cancer-related PE tended to be more asymptomatic and had a lower rate of combined cardiac dysfunction and smaller burden of thromboembolism. However, despite less severe clinical manifestations, PE-related adverse events including recurrent VTE and death from PE were significantly higher in patients with cancer-related PE. The rate of anticoagulation-related bleeding events was similar in the two groups. Our data show the differential clinical manifes- 


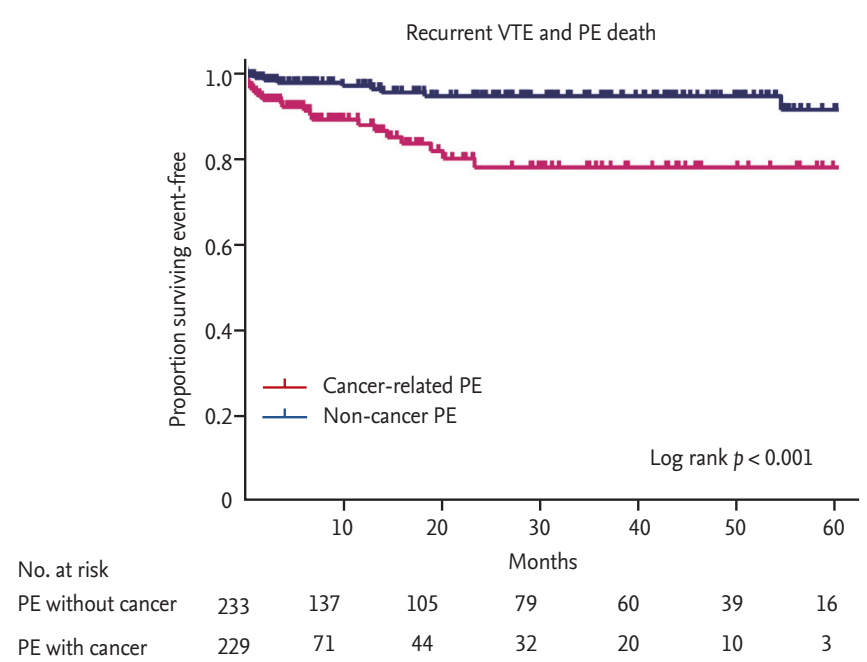

A

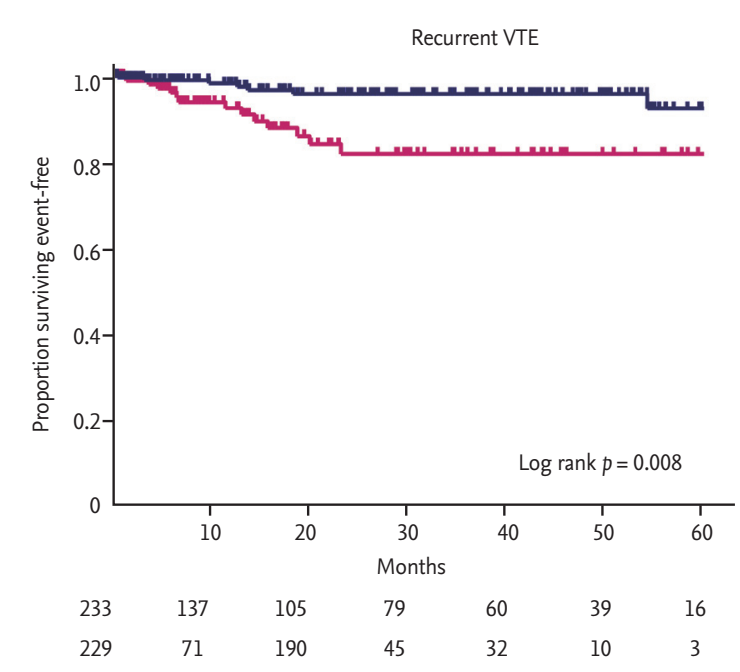

B

Figure 3. Clinical outcome according to the presence of cancer in patients with suspected pulmonary embolism (PE). (A) Kaplan-Meier curve for primary composite outcome between cancer group and non-cancer group. Cancer group showed worse prognosis than non-cancer group (hazard ratio, 13.2; $p=0.003$ ). (B) Kaplan-Meier curve for recurrent venous thromboembolism (VTE) between cancer group and non-cancer group. PE, pulmonary embolism.

tations of cancer-related PE and subsequent PE-related clinical outcomes from our consecutive PE registry data. Although currently recommended treatment strategies for cancer-related $\mathrm{PE}$ are not different than those for non-cancer PE, in terms of real world clinical practice, the treatment rate of $\mathrm{PE}$ is relatively low in patients who present with cancer-related PE compared to patients without cancer [9]. In our data, the treatment rate of cancer-related PE was only $62.0 \%$, which is significantly lower than those diagnosed with other causes of PE. There are several possible explanations for our findings. First, as shown in the results, the clinical features of PE were far less severe with cancer-related PE than with non-cancer PE, despite uncontrolled hypercoagulability related to malignancy and the poor functional status of cancer patients. Second, concern regarding potential interactions between anticoagulants and anticancer drugs or relatively short expected survival may cause physicians to waver in treating PE in patients with cancer. Moreover, the high bleeding risk associated with bone marrow suppression hampers the use of anticoagulants in patients with cancer. The relatively mild clinical manifestations at PE diagnosis and concerns about anticoagulation-related complications are likely the main causes for neglecting to provide adequate treatment of thromboembolism in patients with cancer.
Incidentally detected PE is more frequent in patients with cancer than in those without cancer. However, there is a conflict regarding the efficacy of anticoagulation in incidentally detected PE. While some researchers report that cancer patients with incidental PE have a similar risk for mortality and PE recurrence compared to those with suspected PE, some studies demonstrated that the risk of progressive or recurrent PE without anticoagulation is low in patients with incidental PE [11-13]. For this reason, we also analyzed a subgroup diagnosed with suspected PE. Even in patients with suspected PE, patients with cancer consistently had mild initial clinical features, a low rate of anticoagulation, and higher VTE recurrence than patients without cancer.

Although physicians may feel burdened by anticoagulation therapy for patients with cancer because of concerns about bleeding complications, malignancy does not raise the risk of bleeding in practice. At the time of the study, when before the effectiveness and safety of new oral anticoagulant (NOAC) was confirmed in cancer-related PE patients, the use of NOAC was relatively low in this study. However, only 36 patients treated with anticoagulants experienced major bleeding events in our study. Furthermore, there were no statistically significant differences in bleeding complications between cancer-related PE and non-cancer PE. These observa- 
tions support the current guideline recommendations for treatment with anticoagulation, even in patients with cancer-related PE.

Although the association between smoking and VTE recurrence remains controversial, several studies showed that some factors induced by smoking may promote formation of a clot and subsequent VTE. In our study, smoking was a poor prognostic factor in patients with non-cancer PE. However, with cancer-related PE, there was no significant association between smoking and VTE recurrence. This is because the presence of airflow limitation did not increase the risk of VTE recurrence in cancer patients with $\mathrm{PE}$ [14].

In contrast to previous studies, cancers associated with especially high rates of $\mathrm{PE}$ are not consistent with other research [15-17]. Several western studies showed that pancreatic cancer is associated with a high VTE risk [18]. In this study, however, lung cancer was the most common cancer in patients with cancer-related PE. This may result from differences in the incidence of cancer among races. Lung cancer is the most common cancer in all of Asia [19]. In particular, it is the second most common cancer in Korea. Similarly, a report from China described lung cancer as the most common malignancy diagnosed in patients with PE [20]. With advances in anticancer treatment, especially in patients with lung cancer, expected survival is significantly increasing. Therefore, it is important to treat comorbidities in addition to treating cancer itself. Given the higher rate of recurrent VTE and relatively similar risk of anticoagulation-associated bleeding events in patients with cancer, more active PE treatment is warranted.

\section{Study limitation}

This study has some limitations. Its retrospective design may enhance information bias. To minimize this bias, a prespecified and standardized protocol was used to thoroughly review all medical charts of included patients. Cancer patients may have undergone heightened surveillance for VTE, leading to surveillance bias. Although the risk of VTE varies with different types of cancer [18], this study included all stages of cancer. Further research is needed to describe the characteristics and outcomes of PE according to cancer stage. Median follow-up duration was 10.4 months. The follow-up duration was shorter than expected, which is largely at- tributed to cancer-related deaths.

In conclusion, the treatment rate of PE was $62.0 \%$ in patients with current cancer. In patients with cancer, $\mathrm{PE}$ tended to have less aggressive initial clinical manifestations and thrombus burden compared to PE in patients without cancer. However, given the high rate of recurrent VTE, more active PE treatment is warranted.

\section{KEY MESSAGE}

1. Pulmonary embolism (PE) with cancer was less aggressive initially than those without cancer.

2. PE related adverse events were higher in patients with cancer.

3. In terms of bleeding complication, there were no statistically significant differences between cancer-related PE and non-cancer PE.

\section{Conflict of interest}

No potential conflict of interest relevant to this article was reported.

\section{REFERENCES}

1. Khorana AA, Connolly GC. Assessing risk of venous thromboembolism in the patient with cancer. J Clin Oncol 2009;27:4839-4847.

2. Heit JA, O'Fallon WM, Petterson TM, et al. Relative impact of risk factors for deep vein thrombosis and pulmonary embolism: a population-based study. Arch Intern Med 2002;162:1245-1248.

3. Levine MN, Gent M, Hirsh J, et al. The thrombogenic effect of anticancer drug therapy in women with stage II breast cancer. N Engl J Med 1988;318:404-407.

4. Rodeghiero F, Elice F. Thalidomide and thrombosis. Pathophysiol Haemost Thromb 2003;33 Suppl 1:15-18.

5. Schimmel KJ, Richel DJ, van den Brink RB, Guchelaar HJ. Cardiotoxicity of cytotoxic drugs. Cancer Treat Rev 2004;30:181-191.

6. Albini A, Pennesi G, Donatelli F, Cammarota R, De Flora S, Noonan DM. Cardiotoxicity of anticancer drugs: the need for cardio-oncology and cardio-oncological prevention. J Natl Cancer Inst 2010;102:14-25.

7. Pruthi RK. Review of the American College of Chest Phy- 
sicians 2012 guidelines for anticoagulation therapy and prevention of thrombosis. Semin Hematol 2013;50:251258.

8. Cesarman-Maus G, Ruiz-Arguelles GJ. News in the indications of direct oral anticoagulants according to the American College of Chest Physicians 2016 guidelines. Curr Drug Metab 2017;18:651-656.

9. Sun JM, Kim TS, Lee J, et al. Unsuspected pulmonary emboli in lung cancer patients: the impact on survival and the significance of anticoagulation therapy. Lung Cancer 2010;69:330-336.

10. Schulman S, Kearon C; Subcommittee on Control of Anticoagulation of the Scientific and Standardization Committee of the International Society on Thrombosis and Haemostasis. Definition of major bleeding in clinical investigations of antihemostatic medicinal products in non-surgical patients. J Thromb Haemost 2005;3:692-694.

11. van der Hulle T, den Exter PL, Planquette B, et al. Risk of recurrent venous thromboembolism and major hemorrhage in cancer-associated incidental pulmonary embolism among treated and untreated patients: a pooled analysis of 926 patients. J Thromb Haemost 2016;14:105113.

12. Sahut D'Izarn M, Caumont Prim A, Planquette B, et al. Risk factors and clinical outcome of unsuspected pulmonary embolism in cancer patients: a case-control study. J Thromb Haemost 2012;10:2032-2038.

13. Douma RA, Kok MG, Verberne LM, Kamphuisen PW, Buller HR. Incidental venous thromboembolism in can- cer patients: prevalence and consequence. Thromb Res 2010;125:е306-е309.

14. Shin SH, Kang D, Cho J, et al. Lack of association between airflow limitation and recurrence of venous thromboembolism among cancer patients with pulmonary embolism. Int J Chron Obstruct Pulmon Dis 2018;13:937-943.

15. Blom JW, Vanderschoot JP, Oostindier MJ, Osanto S, van der Meer FJ, Rosendaal FR. Incidence of venous thrombosis in a large cohort of 66,329 cancer patients: results of a record linkage study. J Thromb Haemost 2006;4:529-535.

16. Levitan N, Dowlati A, Remick SC, et al. Rates of initial and recurrent thromboembolic disease among patients with malignancy versus those without malignancy. Risk analysis using Medicare claims data. Medicine (Baltimore) 1999;78:285-291.

17. Khorana AA, Francis CW, Culakova E, Kuderer NM, Lyman GH. Frequency, risk factors, and trends for venous thromboembolism among hospitalized cancer patients. Cancer 2007;110:2339-2346.

18. Chew HK, Wun T, Harvey D, Zhou H, White RH. Incidence of venous thromboembolism and its effect on survival among patients with common cancers. Arch Intern Med 2006;166:458-464.

19. Moore MA, Attasara P, Khuhaprema T, et al. Cancer epidemiology in mainland South-East Asia: past, present and future. Asian Pac J Cancer Prev 2010;11 Suppl 2:67-80.

2o. Wang H, Huang Y, Xu CW, Lin L. Clinical analysis of tumor and non-tumor patients complicated with pulmonary embolism. Int J Clin Exp Med 2015;8:18729-18736. 
Kim MS, et al. Pulmonary embolism in cancer patients

Supplementary Table 1. Baseline characteristics in patients with symptomatic PE

\begin{tabular}{|c|c|c|c|c|}
\hline \multirow{2}{*}{ Variable } & \multicolumn{2}{|c|}{ Suspected PE } & \multirow{2}{*}{$\operatorname{Total}(n=462)$} & \multirow{2}{*}{$p$ value } \\
\hline & Without cancer $(n=233)$ & With cancer $(n=229)$ & & \\
\hline Age, yr & $70(54-77)$ & $64(56-72)$ & $66(54-75)$ & 0.011 \\
\hline Male sex & $101(46.1)$ & $118(51.5)$ & $219(47 \cdot 4)$ & 0.078 \\
\hline $\mathrm{BMI}, \mathrm{kg} / \mathrm{m}^{2}$ & $24.1(21.4-27.1)$ & $23.3(21.2-25.4)$ & $23.7(21.4-26.1)$ & 0.014 \\
\hline Current smoker & $28(12.2)$ & $11(4.8)$ & $39(8.5)$ & 0.005 \\
\hline \multicolumn{5}{|l|}{ Comorbidities } \\
\hline Diabetes & $31(13 \cdot 3)$ & $41(17 \cdot 9)$ & $72(15.6)$ & 0.173 \\
\hline Hypertension & $89(38.2)$ & $78(34.1)$ & $167(36.1)$ & 0.355 \\
\hline Chronic kidney disease & $21(9.0)$ & $18(7 \cdot 9)$ & $39(8.4)$ & 0.656 \\
\hline Previous DVT & $11(4.7)$ & $16(7.0)$ & $27(5.8)$ & 0.299 \\
\hline Previous cancer & $26(11.2)$ & $9(1.9)$ & $35(7 \cdot 6)$ & 0.003 \\
\hline
\end{tabular}

Values are presented as median (interquartile range) or number (\%).

PE, pulmonary embolism; BMI, body mass index; DVT, deep vein thrombosis. 
Supplementary Table 2. Clinical manifestation in patients with symptomatic PE

\begin{tabular}{|c|c|c|c|c|}
\hline \multirow{2}{*}{ Variable } & \multicolumn{2}{|c|}{ Suspected PE } & \multirow{2}{*}{$\operatorname{Total}(n=462)$} & \multirow{2}{*}{$p$ value } \\
\hline & PE without cancer $(n=223)$ & $\mathrm{PE}$ with cancer $(\mathrm{n}=\mathbf{2 2 9})$ & & \\
\hline \multicolumn{5}{|l|}{ Initial symptom/sign } \\
\hline No symptom & $9(3.9)$ & $10(4 \cdot 4)$ & $19(4.1)$ & 0.785 \\
\hline Dyspnea & $136(58.4)$ & $148(64.6)$ & $284(61.5)$ & 0.167 \\
\hline Chest pain & $39(16.7)$ & $24(10.5)$ & $63(13.6)$ & 0.050 \\
\hline Leg swelling & $46(19 \cdot 7)$ & $46(20.1)$ & $92(19.9)$ & 0.926 \\
\hline Fever & $50(21.5)$ & $34(14.8)$ & $84(18.2)$ & 0.065 \\
\hline Hypoxia & $131(57.0)$ & $135(59 \cdot 5)$ & $266(58.2)$ & 0.586 \\
\hline Tachycardia & $108(47.0)$ & $132(58.1)$ & $240(52.5)$ & 0.017 \\
\hline Shock & $35(15.2)$ & $31(13 \cdot 7)$ & $66(14.4)$ & 0.635 \\
\hline \multicolumn{5}{|l|}{ Laboratory finding } \\
\hline D-dimer & $7.1(3 \cdot 3-16.9)$ & $12.6(6.4-23.9)$ & $7.9(2.7-19.1)$ & 0.001 \\
\hline Hemoglobin & $12.4(9.8-13.7)$ & $11.0(9.8-12.5)$ & $11.4(9.8-13.1)$ & $<0.001$ \\
\hline Platelet & $198(147-241)$ & $168(117-224)$ & $191(136-247)$ & $0.05^{8}$ \\
\hline NT-proBNP & $1,235(210-3,847)$ & $636(229-2,119)$ & $741(204-2,920)$ & 0.095 \\
\hline CK-MB & $2.29(0.95-5.48)$ & $1.89(0.69-3.67)$ & $1.89(0.73-4.20)$ & 0.064 \\
\hline Troponin I & $0.043(0.009-0.246)$ & $0.024(0.008-0.125)$ & $0.029(0.007-0.134)$ & 0.207 \\
\hline \multicolumn{5}{|c|}{ Echocardiographic finding } \\
\hline RV dysfunction & $41(30.1)$ & $17(18.7)$ & $58(25.6)$ & 0.052 \\
\hline D-shaped LV & $32(23 \cdot 5)$ & $12(13.2)$ & $44(19 \cdot 4)$ & 0.053 \\
\hline \multicolumn{5}{|l|}{ CT finding } \\
\hline Proximal $^{\mathrm{a}}$ & $149(63.9)$ & $142(62.0)$ & $291(63.0)$ & 0.666 \\
\hline Bilateral involvement & $131(56.2)$ & $123(53.7)$ & $254(55 \cdot 0)$ & 0.587 \\
\hline Lung infarction & $27(11.6)$ & $15(6.6)$ & $42(9.1)$ & 0.060 \\
\hline \multicolumn{5}{|l|}{ Coexisting $\mathrm{DVT}^{\mathrm{b}}$} \\
\hline Distal DVT & $40(19 \cdot 4)$ & $34(19.2)$ & $74(19 \cdot 3)$ & 0.979 \\
\hline Proximal DVT & $84(40.8)$ & $74(41.8)$ & $158(41.3)$ & 0.979 \\
\hline
\end{tabular}

Values are presented as number (\%) or median (interquartile range).

PE, pulmonary embolism; NT-proBNP, N-terminal pro-blood natriuretic peptide; CK-MB, creatine kinase myocardial band; $\mathrm{RV}$, right ventricle, LV, left ventricle; CT, computed tomography; DVT, deep vein thrombosis.

${ }^{a}$ Proximal PE includes filling defects in the main, central and lobar pulmonary artery.

${ }^{\mathrm{b}}$ DVT is confirmed using Doppler ultrasonography or computed tomography. Proximal DVT was defined as occurring in the popliteal vein and above. 
Kim MS, et al. Pulmonary embolism in cancer patients

Supplementary Table 3. Predictive factors for recurrent VTE and death from PE

\begin{tabular}{llll}
\hline Variable & Adjusted HR & 95\% CI & palue \\
\hline All patients with PE & & & 0.001 \\
$\quad$ Cancer & 9.75 & $2.4-39.2$ & 0.048 \\
\hline No anticoagulation & 3.95 & $1.1-11.8$ & 0.006 \\
\hline Current smoking & 5.3 & $1.6-17.3$ & 0.048 \\
\hline Bilateral lung involvement & 3.46 & $1.0-11.8$ & 0.045 \\
\hline Incidental PE & 0.1 & $0.0-0.95$ & 0.004 \\
Suspected PE & & & 0.002 \\
\hline Cancer & 8.70 & $2.0-37.4$ & 0.004 \\
\hline No anticoagulation & 7.96 & $2.1-30.5$ & $1.8-20.1$ \\
\hline Current smoking & 5.94 & & \\
\hline
\end{tabular}

Adjusted for age, gender, body mass index, cancer, no anticoagulation, duration of treatment, proximal located PE, bilateral lung involvement, shock, tachycardia, hypoxia, smoking, right ventricular dysfunction, unexpected PE, N-terminal pro-blood natriuretic peptide, creatine kinase myocardial band, hemoglobin, and D-dimer.

VTE, venous thromboembolism; PE, pulmonary embolism; HR, hazard ratio; CI, confidence interval. 